\title{
THE TRANSFER IDEAL OF QUADRATIC FORMS AND A HASSE NORM THEOREM MOD SQUARES
}

\author{
DAVID B. LEEP AND ADRIAN R. WADSWORTH
}

\begin{abstract}
Any finite degree field extension $K / F$ determines an ideal $\mathscr{T}_{K / F}$ of the Witt ring $W F$ of $F$, called the transfer ideal, which is the image of any nonzero transfer map $W K \rightarrow W F$. The ideal $\mathscr{T}_{K / F}$ is computed for certain field extensions, concentrating on the case where $K$ has the form $F\left(\sqrt{a_{1}}, \ldots, \sqrt{a_{n}}\right), \quad a_{i} \in F$. When $F$ and $K$ are global fields, we investigate whether there is a local global principle for membership in $\mathscr{T}_{K / F}$. This is shown to be equivalent to the existence of a "Hasse norm theorem mod squares," i.e., a local global principle for the image of the norm map $N_{K / F}: K^{*} / K^{* 2} \rightarrow F^{*} / F^{* 2}$. It is shown that such a Hasse norm theorem holds whenever $K=F\left(\sqrt{a_{1}}, \ldots, \sqrt{a_{n}}\right)$, although it does not always hold for more general extensions of global fields, even some Galois extensions with group $\mathbf{Z} / \mathbf{Z} \mathbf{Z} \times \mathbf{Z} / \mathbf{4} \mathbf{Z}$.
\end{abstract}

\section{INTRODUCTION}

For any field $F$ with $\operatorname{char}(F) \neq 2$, let $W F$ denote the Witt ring of quadratic forms over $F$. If $K \supseteq F$ is another field, with $[K: F]<\infty$, and if $s: K \rightarrow F$ is any nonzero $F$-linear map, then there is an induced Scharlau transfer map $s_{*}: W K \rightarrow W F$. Since $s_{*}$ is $W F$-linear, its image is an ideal of $W F$. This ideal is known to depend only on $K$ and $F$ and not on the choice of $s$ (cf., [S, p. 48, Corollary 5.7]); we call it the transfer ideal of $K$ over $F$, denoted $\mathscr{T}_{K / F}$. Since so many arguments in quadratic form theory involve passage back and forth between a field and nearby fields it is of interest to determine $\mathscr{T}_{K / F}$ whenever possible. Yet, beyond the well-known formula when $K=F(\sqrt{a})$ (then $\mathscr{I}_{K / F}=\operatorname{ann}_{W F}\langle 1,-a\rangle$ ), very little work seems to have been done on $\mathscr{T}_{K / F}$. While it seems hopeless to determine $\mathscr{T}_{K / F}$ in general, there are some important families of field extensions for which we will describe $\mathscr{T}_{K / F}$ quite completely.

There are two particular questions, raised by D. Shapiro, which motivated the work presented here. They are:

1. If $K=F\left(\sqrt{a_{1}}, \ldots, \sqrt{a_{n}}\right)$ with each $a_{i} \in F$ (i.e., $K$ is a multiquadratic extension of $F$ ) is $\mathscr{T}_{K / F}=\bigcap_{i=1}^{n} \mathscr{T}_{F\left(\sqrt{a_{i}}\right) / F}$ ?

Received by the editors April 28, 1989.

1980 Mathematics Subject Classification (1985 Revision). Primary 11E04, 11R20.

The second author was supported in part by the National Science Foundation. 
This would be a natural generalization of the basic case of $K=F(\sqrt{a})$. Unfortunately, the answer to this is no in general for $n \geq 3$ (though yes for $n=2)$. We show in $\S 2$ that the validity of

$$
\mathscr{T}_{F\left(\sqrt{a_{1}}, \sqrt{a_{2}}, \sqrt{a_{3}}\right) / F}=\bigcap_{i=1}^{3} \mathscr{T}_{F\left(\sqrt{a_{i}}\right) / F}
$$

for any $a_{1}, a_{2}, a_{3} \in F$ is equivalent to $F$ satisfying the property $P_{1}(3)$ studied in [STW], and to $F$ satisfying the property $C V(2,2)$ of [SL]. The known counterexamples to these properties thus provide counterexamples to Question 1. On the positive side, we show in $\S \S 3-5$ that the answer to Question 1 is yes if $F$ is a local or a global field, or a field with Hasse number $\tilde{u}(F) \leq 2$.

2. Is there a local-global principle for $\mathscr{T}_{K / F}$ when $F \subseteq K$ are global fields?

Since it is not difficult to describe $\mathscr{T}_{K / F}$ for $F \subseteq K$ local fields (we do this in $\S 3)$, such a local-global principle would provide a reasonable way to determine $\mathscr{T}_{K / F}$ for global fields. We show in $\S 1$ that for certain fields including local and global fields $\mathscr{T}_{K / F}$ is essentially determined by the image of the norm map mod squares: $N_{K / F}: K^{*} / K^{* 2} \rightarrow F^{*} / F^{* 2}$. Thus, Question 2 becomes equivalent to the question whether there is a local global principle for the image of $N_{K / F}$; such a result would be a "Hasse norm theorem mod squares." We prove in $\S 4$ that the answer to Question 2 is yes if $K$ is a multiquadratic extension of $F$. Thus, for such an extension the Hasse norm theorem mod squares holds. This is somewhat remarkable since it is well known that the usual Hasse norm theorem does not hold in general even for biquadratic extensions. However, it will be shown in [LW] that Hasse mod squares does not hold for all extensions of global fields, including some with Galois group $\mathbb{Z} / 2 \mathbb{Z} \times \mathbb{Z} / 4 \mathbb{Z}$. Such extensions yield counterexamples to Question 2.

We thank D. Shapiro for stimulating our interest in the transfer ideal, and for asking us Questions 1 and 2.

We will generally follow the notation in [L] and [S] for quadratic form theory. Thus, if $F$ is a field (always assumed of characteristic not 2) $W F$ denotes the Witt ring of quadratic forms over $F ; I F$ is the fundamental ideal of evendimensional forms in $W F$; and $I^{n} F$ denotes the $n$th power $(I F)^{n}$ of $I F$. We write $\left\langle a_{1}, \ldots, a_{n}\right\rangle$ for the $n$-dimensional diagonal quadratic form $a_{1} x_{1}^{2}+$ $\cdots+a_{n} x_{n}^{2}$, and $\left\langle\left\langle a_{1}, \ldots, a_{n}\right\rangle\right\rangle$ for the $n$-fold Pfister form $\left\langle 1, a_{1}\right\rangle \otimes \cdots \otimes\left\langle 1, a_{n}\right\rangle$. The multiplicative group $F-\{0\}$ of $F$ is denoted $F^{*}$. If $q$ is a quadratic form over $F$, we write $D_{F}(q)$ for the set of values in $F^{*}$ represented by $q$.

\section{GENERAL PROPERTIES OF THE TRANSFER IDEAL}

Let $K / F$ be a finite degree extension of fields, $\operatorname{char}(F) \neq 2$, and let $\mathscr{T}_{K / F}$ be the transfer ideal of $W F$ defined in the Introduction. Our main goal in this paper is to calculate $\mathscr{T}_{K / F}$ in several interesting cases and we begin by defining some related objects. 


\section{Definition 1.1.}

(1) Let $N_{K / F}: K^{*} / K^{* 2} \rightarrow F^{*} / F^{* 2}$ be the group homomorphism induced by the usual norm map from $K^{*}$ to $F^{*}$. (For convenience, if $\alpha \in K^{*}$, we set $N_{K / F}(\alpha)=N_{K / F}\left(\alpha K^{* 2}\right)$, and write $N_{K / F}(\alpha)=a \in F^{*}$ to mean $N_{K / F}(\alpha)=a F^{* 2}$.)

(2) Let $G_{K / F}=\operatorname{im}\left(N_{K / F}\right)$ in $F^{*} / F^{* 2}$.

(3) Let $\mathfrak{A}\left(G_{K / F}\right)$ be the ideal in $W F$ generated by all binary forms $\langle 1,-g\rangle$ where $g F^{* 2} \in G_{K / F}$.

(4) Let $i_{K / F}: W F \rightarrow W K$ be the canonical ring homomorphism induced by the inclusion $F \hookrightarrow K$. Let $W(K / F)$ be the kernel of $i_{K / F}$, so $W(K / F)$ is an ideal of $W F$. Let ann $W(K / F)$ be the annihilator of $W(K / F)$ in $W F$.

Lemma 1.2. If $[K: F]$ is odd then $G_{K / F}=F^{*} / F^{* 2}, \mathfrak{A}\left(G_{K / F}\right)=I F$, and $\mathscr{T}_{K / F}=\operatorname{ann} W(K / F)=W F$.

Proof. If $a \in F^{*}$, then $N_{K / F}(a)=a^{[K: F]} F^{* 2}=a F^{* 2}$. Therefore, $G_{K / F}=$ $F^{*} / F^{* 2}$ and $\mathfrak{A}\left(G_{K / F}\right)=I F$. By Springer's theorem $W(K / F)=0$, and hence ann $W(K / F)=W F$. Finally $\mathscr{T}_{K / F}=W F$, by [L, p. 195, Theorem 1.6(2)].

Lemma 1.3. If $[K: F]$ is even, then $s_{*}(W K)=s_{*}(I K)$ for any nonzero $F$-linear map $s: K \rightarrow F$.

Proof. We can assume $K$ is a simple extension of $F$. If $K=F(\theta)$ there is a transfer $s_{*}: W K \rightarrow W F$ with $s_{*}(\langle\theta\rangle)=0$ (see [L, p. 196, Theorem 1.7(1)]). Then for any $q \in W K, s_{*}(q)=s_{*}(q \perp\langle\theta\rangle)$, showing $s_{*}(W K)=s_{*}(I K)$.

Proposition 1.4. $\mathfrak{A}\left(G_{K / F}\right) \subseteq \mathscr{T}_{K / F} \subseteq$ ann $W(K / F)$.

Proof. Take any $q \in W(K / F)$ and $q^{\prime} \in \mathscr{T}_{K / F}$, say $q^{\prime}=s_{*}(\tau)$ for $\tau \in W K$. Then by the Frobenius reciprocity for the transfer, $q \cdot q^{\prime}=s_{*}\left(q_{K} \tau\right)=s_{*}(0)=0$. Thus, $\mathscr{T}_{K / F} \subseteq$ ann $W(K / F)$.

To show $\mathfrak{A}\left(G_{K / F}\right) \subseteq \mathscr{T}_{K / F}$ it suffices to verify $\langle 1,-g\rangle \in \mathscr{T}_{K / F}$ whenever $g F^{* 2} \in G_{K / F}$. We may assume $[K: F]$ is even by Lemma 1.2. We have $g=N_{K / F}(\alpha)$ for some $\alpha \in K^{*}$. Let $E=F(\alpha)$, and let $r=[K: E]$. Then $g=N_{E / F}(\alpha)^{r}$. If $r$ is even, then $g \in F^{* 2}$ and $\langle 1,-g\rangle=0 \in \mathscr{T}_{K / F}$. Assume $r$ is odd, so $[E: F]$ is even. Then $\mathscr{T}_{K / F}=\mathscr{T}_{E / F}$ by Lemma 1.2. So, using [L, p. 195, Theorem 1.6(1)] $\langle 1,-g\rangle \cong\left\langle 1,-N_{E / F}(\alpha)\right\rangle \in \mathscr{T}_{E / F}=\mathscr{T}_{K / F}$, as desired.

The behavior of the transfer ideal under scalar extensions is known: 
Lemma 1.5. Let $F \subseteq K, L$ be fields with $[K: F]<\infty$. Then,

(1) If $K \otimes_{F} L \cong E_{1} \oplus \cdots \oplus E_{n}$, with each $E_{i}$ a field, then $i_{L / F}\left(\mathscr{T}_{K / F}\right) \subseteq$ $\sum_{i} \mathscr{T}_{E_{i} / L}$

(2) If $K$ is Galois over $F$ and $K \cdot L$ is any compositum of $K$ and $L$ over $F$, then $i_{L / F}\left(\mathscr{T}_{K / F}\right) \subseteq \mathscr{T}_{K \cdot L / L}$.

Proof. (1) is immediate from [A, Satz 2.2]. Then (2) follows from (1), since when $K$ is Galois over $F$ each $E_{i}$ is $L$-isomorphic to $K \cdot L$.

For a quadratic form $q$ over $F$, let $d_{ \pm}(q)$ be the signed determinant of $q$, i.e., $d_{ \pm}(q)=(-1)^{n(n-1) / 2} \operatorname{det}(q) \in F^{*} / F^{* 2}$, where $n=\operatorname{dim} q$.

Lemma 1.6. For any nonzero $F$-linear map $s: K \rightarrow F$ the following diagram is commutative:

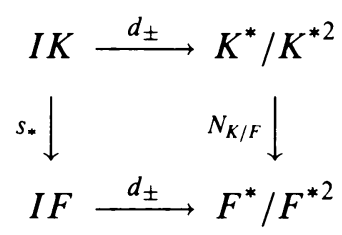

Proof. See [S, p. 51, Theorem 5.12].

Lemma 1.7. Take any $q \in W F$ and $g \in F^{*}$.

(1) If $q \in \mathscr{T}_{K / F}$ then $d_{ \pm}(q) \in G_{K / F}$.

(2) $\langle 1,-g\rangle \in \mathscr{T}_{K / F}$ if and only if $g F^{* 2} \in G_{K / F}$.

(3) Suppose $q \in I F$, and suppose $q=q_{1} \perp q_{2}$, where $\operatorname{dim} q_{1}=2$ and $q_{2} \in I^{2} F$. Then $q \in \mathscr{T}_{K / F}$ if and only if $q_{1} \in \mathfrak{A}\left(G_{K / F}\right)$ and $q_{2} \in \mathscr{T}_{K / F}$.

(4) If $[K: F]$ is even, then $\mathscr{T}_{K / F}=\mathfrak{A}\left(G_{K / F}\right)+\left(\mathscr{T}_{K / F} \cap I^{2} F\right)$.

Proof. (1) follows easily from, Lemmas 1.2, 1.3, and 1.6. Then (2) follows by (1) and Proposition 1.4.

(3) Suppose $q \in \mathscr{T}_{K / F}$. Then $d_{ \pm}\left(q_{1}\right)=d_{ \pm}(q) \in G_{K / F}$ by (1). So, $q_{1}=$ $c\left\langle 1,-d_{ \pm}(q)\right\rangle \in \mathscr{T}_{K / F}$ by (2), and $q_{2}=q-q_{1} \in \mathscr{T}_{K / F}$. Conversely, if $q_{1} \in$ $\mathfrak{A}\left(G_{K / F}\right)$ and $q_{2} \in \mathscr{T}_{K / F}$, then $q \in \mathscr{T}_{K / F}$ by Proposition 1.4.

(4) This is immediate from (3) and Proposition 1.4, since every $q \in I F$ can be decomposed as $q=\langle 1, a\rangle \perp q_{2}$ where $q_{2} \in I^{2} F$.

The description of the transfer ideal for a quadratic extension is well known:

Proposition 1.8. Let $K=F(\sqrt{a})$ for $a \in F^{*}$, with $[K: F]=2$. Then $G_{K / F}=$ $D_{F}\langle 1,-a\rangle / F^{* 2}, W(K / F)=W F\langle 1,-a\rangle$ and $\mathfrak{A}\left(G_{K / F}\right)=\mathscr{T}_{K / F}=$ ann $W(K / F)$.

Proof. The formula for the norm yields $G_{K / F}=D_{F}\langle 1,-a\rangle / F^{* 2}$. The exact triangle for a quadratic extension [S, p. 50] determines $W(K / F)$ and shows $\mathscr{T}_{K / F}=$ ann $W(K / F)$. Since the annihilator of a Pfister form is generated by 
2-dimensional forms [S, p. 74, Theorem 10.13], Lemma 1.7(3) shows $\mathscr{T}_{K / F}=$ $\mathfrak{A}\left(G_{K / F}\right)$.

Proposition 1.9. Let $K=F(\sqrt{a}, \sqrt{b})$ for $a, b \in F^{*}$, with $[K: F]=4$. Then,

(1) $G_{K / F}=\operatorname{im}\left(N_{F(\sqrt{a}) / F}\right) \cap \operatorname{im}\left(N_{F(\sqrt{b}) / F}\right)=\left(D_{F}\langle\langle-a\rangle\rangle \cap D_{F}\langle\langle-b\rangle\rangle\right) / F^{* 2}$.

(2) $\mathscr{T}_{K / F}=\operatorname{ann} W(K / F)=\mathscr{T}_{F(\sqrt{a}) / F} \cap \mathscr{T}_{F(\sqrt{b}) / F}=\operatorname{ann}\langle\langle-a\rangle\rangle \cap \operatorname{ann}\langle\langle-b\rangle\rangle$.

The ideal $\mathscr{T}_{K / F}$ is generated by 1-fold and 2-fold Pfister forms.

(3) Although $\mathfrak{A}\left(G_{K / F}\right) \subseteq \mathscr{T}_{K / F}$, equality need not hold.

Proof. The second equality of (2) holds since

$$
W(K / F)=\langle\langle-a\rangle\rangle W F+\langle\langle-b\rangle\rangle W F
$$

by [ELW , Corollary 2.12]. The first equality of (2) then follows by [E, Proposition 2.5]. (In applying [E, Proposition 2.5], take $K=F(\sqrt{a}), \gamma=\langle\langle-b\rangle\rangle$ and note that ann $\left.{ }_{W K}\left(\gamma_{K}\right)=\mathscr{T}_{F(\sqrt{a}, \sqrt{b}) / F(\sqrt{a})}.\right)$ It is shown in $\left[\mathrm{ELW}_{2}\right.$, Corollary 4.9] that ann $\langle\langle-a\rangle\rangle$ กann $\langle\langle-b\rangle\rangle$ is generated by 1 -fold and 2-fold Pfister forms. The equalities in (1) follow from (2), Lemma 1.7(2), and Proposition 1.8.

To show (3), let $F$ be any nondyadic local field (so $\left|F^{*} / F^{* 2}\right|=4$ ) and let $K$ be the unique biquadratic extension of $F$. Then $\left|G_{K / F}\right|=1$ (see the proof of Theorem 3.2 below), so $\mathfrak{A}\left(G_{K / F}\right)=(0)$. But the anistropic 2-fold Pfister form $\varphi$ of $F$ lies in $\mathscr{T}_{K / F}$ since $I^{3} F=0$ implies $\varphi \in \operatorname{ann}(I F) \subseteq \mathscr{T}_{K / F}$.

Consideration of more general multiquadratic extensions will be deferred to $\S 2$.

Lemma 1.7 reduces the determination of $\mathscr{T}_{K / F}$ to computing $G_{K / F}$ and $\mathscr{T}_{K / F} \cap I^{2} F$. We will be interested in cases where $\mathscr{T}_{K / F} \cap I^{2} F$ can be computed by signature conditions relative to the orderings of $F$. Let $X_{F}$ denote the (compact, Hausdorff) topological space of orderings of $F$ (see [S, Chapter 3, $\S 5])$. For any field $K \supseteq F$, let $\varepsilon_{K / F}: X_{K} \rightarrow X_{F}$ denote the (continuous) function mapping an ordering of $K$ to its restriction to $F$. If $P \in X_{F}$, then $<_{P}$ denotes the associated order relation of $F$, so $P=\left\{a \in F \mid a \geq_{P} 0\right\}$. For $q \in W F, \operatorname{sgn}_{P}(q)$ denotes the signature of $q$ relative to $P$.

The following theorem is given (in a somewhat more general form) in the thesis of R. Bos [Bo, p. 139, Theorem 6.9], which regrettably is unpublished at this time. We therefore give a proof, adapted from the one in [Bo]. We thank R. Bos for his kind permission to use his argument.

Theorem 1.10 (Bos). Let $F \subseteq K$ be formally real fields with $[K: F]<\infty$. Then there is a clopen subset $Y$ of $X_{K}$ with $Y$ homeomorphic (via $\varepsilon_{K / F}$ ) to $\operatorname{im}\left(\varepsilon_{K / F}\right)$.

Proof. Let $A=\operatorname{im}\left(\varepsilon_{K / F}\right)$. Then $A$ is a clopen subset of $X_{F}$ since $\varepsilon_{K / F}$ is both a closed mapping, as $X_{K}$ is compact, and also an open mapping by [ELW ${ }_{3}$, Theorem 4.9], as $K$ is finitely-generated over $F$. Take any $P \in A$, and let 
$Q_{1}, \ldots, Q_{k}$ be the extensions of $P$ to $K$. The number of extensions is finite, since it is bounded by $[K: F]$. Since $\left\{Q_{2}, \ldots, Q_{k}\right\}$ is closed in $X_{K}$, there is a neighborhood $B$ of $Q_{1}$ with $Q_{i} \notin B$ for $i \geq 2$. By choosing $B$ to be in the Harrison base of $X_{K}$, we may assume that $B$ is clopen. Then the characteristic function of $B, \chi_{B}: X_{K} \rightarrow\{0,1\}$, is continuous. Let $C(X, Y)$ denote the set of continuous functions from $X$ to $Y$. By [ELTW, (6.6), (6.8)] the trace map $\operatorname{tr}_{K / F}$ induces a function $\operatorname{tr}_{*}: C\left(X_{K}, \mathbb{Z}\right) \rightarrow C\left(X_{F}, \mathbb{Z}\right)$ given by

$$
\operatorname{tr}_{*}(g)\left(P^{\prime}\right)=\sum_{Q \in \varepsilon_{K / F}^{-1}\left(P^{\prime}\right)} g(Q)
$$

for every $P^{\prime}$ in $X_{F}$. Let $f=\operatorname{tr}_{*}\left(\chi_{B}\right) \in C\left(X_{F}, \mathbb{Z}\right)$. Then for all $P^{\prime}, f\left(P^{\prime}\right)=$ $\left|B \cap \varepsilon_{K / F}^{-1}\left(P^{\prime}\right)\right|$, so in particular $f(P)=1$. Let $C_{P}=\varepsilon_{K / F}^{-1}\left(f^{-1}(1)\right) \cap B$, a clopen subset of $X_{K}$ containing $Q_{1}$, and let $D_{P}=\varepsilon_{K / F}\left(C_{P}\right)$. Then $P \in D_{P}$ and $\varepsilon_{K / F}$ maps $C_{P}$ bijectively (hence homeomorphically as $\varepsilon_{K / F}$ is closed) onto $D_{P}$.

Because $A$ is compact the clopen cover $\left\{D_{P} \mid P \in A\right\}$ of $A$ has a finite subcover $D_{P_{1}}, \ldots, D_{P_{n}}$. Let $T_{i}=D_{P_{i}}-\bigcup_{j=1}^{i-1} D_{P_{j}}$, and let $S_{i}=\varepsilon_{K / F}^{-1}\left(T_{i}\right) \cap C_{P_{i}}$, for $i=1,2, \ldots, n$. Then $T_{1}, T_{2}, \ldots, T_{n}$ form a clopen cover of $A$ by disjoint sets. Further, each $S_{i}$ is clopen in $X_{K}$ and is mapped by $\varepsilon_{K / F}$ homeomorphically onto $T_{i}$. Hence, $\bigcup_{i=1}^{n} S_{i}$ is a clopen subset of $X_{K}$ mapping homeomorphically onto $A$, as desired.

Remark. It is shown in [Er] that if $L$ is any finitely-generated (not necessarily algebraic) field extension of $F$, then there is a continuous section map $\sigma: X_{F} \rightarrow X_{L}$ such that $\varepsilon_{L / F} \circ \sigma=\mathrm{id}$. This theorem is also proved in [Bo, p. 140], without the assumption of finite generation. The result in [Er] is insufficient for us here as we need that there is a section $\sigma$ with $\operatorname{im}(\sigma)$ clopen. This is true when $[L: F]<\infty$, but not in general.

For a field $K$, let $s t_{r} K$ denote the reduced stability index of $K$. That is, $s t_{r} K$ is the least integer $n$ such that $I^{n+1} K \subseteq 2 I^{n} K+W_{t} K$, where $W_{t} K$ is the torsion subgroup of $W K$. It is known (cf. [Br, Satz 3.17] and [ELTW, Lemma 8.3]) that $s t_{r} K$ is also the least $n$ such that the total signature map sgn: $I^{n} K \rightarrow C\left(X_{K}, 2^{n} \mathbb{Z}\right)$ is surjective. Recall that $s t_{r} K \leq 1$ if and only if $K$ satisfies SAP [Br, Satz 3.20]. Also, if $K$ is not formally real $s t_{r} K=0$.

Consider the following hypothesis on a field $F$ and an integer $n$ :

$$
\text { For every finite degree extension } K \text { of } F \text {, }
$$

$$
I^{n+1} K \text { is torsion-free and } s t_{r} K \leq n \text {. }
$$

The fields satisfying ( $*$ ) for $n=1$ or $n=2$ are those for which we can describe $\mathscr{T}_{K / F} \cap I^{2} F$ by signature conditions.

Theorem 1.11. Let $F$ be a field satisfying (*) for some integer $n$, and let $K$ be any finite-degree extension of $F$. Then for any transfer $s_{*}^{K / F}: W K \rightarrow W F$, 
$s_{*}^{K / F}\left(I^{n} K\right)=I^{n} F \cap \mathscr{T}_{K / F}=\left\{q \in I^{n} F \mid \operatorname{sgn}_{P}(q)=0\right.$ for each $P \in X_{F}$ which does not extend to $K\}$.

Proof. Observe that $s_{*}^{K / F}\left(I^{n} K\right)$ is the same for any transfer map; at times a specific choice of $s_{*}^{K / F}$ will be convenient. The inclusion $s_{*}^{K / F}\left(I^{n} K\right) \subseteq I^{n} F \cap \mathscr{T}_{K / F}$ follows from [A, Satz 3.3]. The second inclusion $\subseteq$ follows from Knebusch's formula [S, p. 124, Theorem 4.5] which says that for any $q \in W K$, if $s_{*}^{K / F}$ is the trace transfer, then for any $P \in X_{F}$

$$
\operatorname{sgn}_{P}\left(s_{*}^{K / F}(q)\right)=\sum_{Q \in \varepsilon_{K / F}^{-1}(P)} \operatorname{sgn}_{Q}(q) .
$$

To show that the third set lies in $s_{*}^{K / F}\left(I^{n} K\right)$, first assume $F$ is nonreal. Then we must show $I^{n} F \subseteq s_{*}^{K / F}\left(I^{n} K\right)$. It suffices to replace $K$ by $M$ where $M$ is the normal closure of $K$ over $F$. By Galois theory, $M$ is obtainable from $F$ by an odd degree extension followed by a succession of quadratic extensions (followed by another odd degree extension if $M$ is not separable over $F$ ). Since Lemma 1.2 disposes of the odd degree extensions, we can reduce to the case of a quadratic extension $L / E$, where $M \supseteq L \supseteq E \supseteq F$, say $L=E(\sqrt{c})$. We have $I^{n} E \subseteq$ ann $_{W E}\langle\langle-c\rangle\rangle=\mathscr{T}_{L / E}$ since $I^{n+1} E=0$ by $(*)$. It follows that $I^{n} E \subseteq s_{*}^{L / E}\left(I^{n} L\right)$, since every $n$-fold Pfister form in $\mathscr{T}_{L / E}$ is the transfer of an $n$-fold Pfister form from $L$, by [A, p. 459, Zusatz].

Now assume $F$ is formally real. Let $\left(I^{n} F\right)_{t}$ denote the torsion ideal of $I^{n} F$. To show $\left(I^{n} F\right)_{t} \subseteq s_{*}^{K / F}\left(I^{n} K\right)$ it suffices to verify

$$
\left(I^{n} F\right)_{t} \subseteq s_{*}^{K(\sqrt{-1}) / F}\left(I^{n} K(\sqrt{-1})\right) .
$$

For this, note that $I^{n} F(\sqrt{-1}) \subseteq s_{*}^{K(\sqrt{-1}) / F(\sqrt{-1})}\left(I^{n} K(\sqrt{-1})\right)$ by the nonreal case above. In addition, we have $\left(I^{n} F\right)_{t} \subseteq \operatorname{ann}_{W F}\langle\langle 1\rangle\rangle=\mathscr{T}_{F(\sqrt{-1}) / F}$ as $I^{n+1} F$ is torsion-free. But $\left(I^{n} F\right)_{t}$ is generated by torsion Pfister forms. (This holds when $n=1$ or $n=2$ by [EL ${ }_{1}$, Theorem 2.8]. For $n>2$ it holds as $s t_{r} F \leq n$, by $\left[\mathrm{K}\right.$, Theorem 1].) Therefore, it follows as in the nonreal case that $\left(I^{n} F\right)_{t} \subseteq$ $s_{*}^{F(\sqrt{-1}) / F}\left(I^{n} F(\sqrt{-1})\right)$. Thus,

$$
\left(I^{n} F\right)_{t} \subseteq s_{*}^{K(\sqrt{-1}) / F}\left(I^{n} K(\sqrt{-1})\right) \subseteq s_{*}^{K / F}\left(I^{n} K\right) .
$$

To complete the formally real case, let $Y$ be a clopen subset of $X_{K}$ with $Y$ homeomorphic to $\operatorname{im}\left(\varepsilon_{K / F}\right)$, as described in Theorem 1.10. Take any $q \in$ $I^{n} F$ such that $\operatorname{sgn}_{P}(q)=0$ for all $P \in X_{F}-\operatorname{im}\left(\varepsilon_{K / F}\right)$. For $i \in \mathbb{Z}$, let $C_{i}=\left\{P \in X_{F} \mid \operatorname{sgn}_{P}(q)=i 2^{n}\right\}$. Then each $C_{i}$ is a clopen subset of $X_{F}$ and for $N$ sufficiently large, $X_{F}=\bigcup_{i=-N}^{N} C_{i}$, a disjoint union. For $i \neq 0$, set $D_{i}=\varepsilon_{K / F}^{-1}\left(C_{i}\right) \cap Y$, a clopen subset of $X_{K}$. The hypothesis on $q$ assures that $\varepsilon_{K / F}\left(D_{i}\right)=C_{i}$. Since $s t_{r} K \leq n$, there exists for each $i \neq 0, \tau_{i} \in I^{n} K$ 
satisfying

$$
\operatorname{sgn}_{Q}\left(\tau_{i}\right)= \begin{cases}i 2^{n}, & \text { if } Q \in D_{i}, \\ 0, & \text { if } Q \notin D_{i} .\end{cases}
$$

Set $\tau_{0}=0$. Then, (assuming $s_{*}^{K / F}$ is the trace transfer) formula (1.12) yields

$$
\operatorname{sgn}_{P}\left(s_{*}^{K / F}\left(\tau_{i}\right)\right)= \begin{cases}i 2^{n}, & \text { if } P \in C_{i}, \\ 0, & \text { if } P \notin C_{i} .\end{cases}
$$

Let $\tau=\perp_{-N \leq i \leq N} \tau_{i} \in I^{n} K$. Then $\operatorname{sgn}_{P}\left(s_{*}^{K / F}(\tau)\right)=\operatorname{sgn}_{P}(q)$ for all $P \in X_{F}$. By Pfister's local global principle [S, p. 56], $q \perp-s_{*}^{K / F}(\tau) \in\left(I^{n} F\right)_{t} \subseteq s_{*}^{K / F}\left(I^{n} K\right)$. Hence $q \in s_{*}^{K / F}\left(I^{n} K\right)$, completing the proof.

Theorem 1.13. Let $K$ be a finite degree extension of a field $F$ satisfying (*) for $n=1$ or $n=2$. Let $q \in I F$. Then $q \in \mathscr{T}_{K / F}$ if and only if both (1) and (2) hold:

(1) $d_{ \pm}(q) \in G_{K / F}$.

(2) $\operatorname{sgn}_{P}(q)=0$ for all orderings $P \in X_{F}$ which do not extend to $K$.

Furthermore, if $F$ satisfies (*) with $n=1$, then $q \in \mathscr{T}_{K / F}$ if and only if (2) holds.

Proof. If $q \in \mathscr{T}_{K / F}$, then (1) holds by Lemma 1.7(1) and (2) holds by formula (1.12).

Now assume (1) and (2) hold. Since $d_{ \pm}(q) \in G_{K / F}$, we have $\left\langle 1,-d_{ \pm}(q)\right\rangle \in$ $\mathfrak{A}\left(G_{K / F}\right) \subseteq \mathscr{T}_{K / F}$. Therefore, for every $P \in X_{F}$ which does not extend to $K$, $\operatorname{sgn}_{P}\left\langle 1,-d_{ \pm}(q)\right\rangle=0$ by formula (1.12). Let $q^{\prime}=\left\langle 1,-d_{ \pm}(q)\right\rangle \perp q \in I^{2} F$. Then $\operatorname{sgn}_{P}\left(q^{\prime}\right)=\operatorname{sgn}_{P}(q)=0$ for all $P \in X_{F}-\operatorname{im}\left(\varepsilon_{K / F}\right)$. So, Theorem 1.11 implies $q^{\prime} \in \mathscr{T}_{K / F}$. Since $\left\langle 1,-d_{ \pm}(q)\right\rangle \in \mathscr{T}_{K / F}$, it follows that $q \in \mathscr{T}_{K / F}$.

Now assume $F$ satisfies $(*)$ with $n=1$. Then Theorem 1.11 shows $q \in$ $\mathscr{T}_{K / F}$ if (2) holds.

Recall that a finite degree extension of fields $K / F$ is called a 2-extension if $K$ is obtainable from $F$ by a succession of extensions of degree 2 .

Corollary 1.14. Suppose $F$ is a field with $I^{n+1} F$ torsion free and $s t_{r}(F) \leq n$, for $n=1$ or $n=2$. Then the conclusions of Theorems 1.11 and 1.13 hold if $K$ is any 2-extension of $F$.

Proof. We first observe that for $n=1,2$ the properties $(\alpha) s t_{r} F \leq n$ and $I^{n+1} F$ torsion-free are equivalent to $(\beta)$ st $F \leq n$ and $F$ satisfies $A_{n+1}$. (The absolute stability index $s t_{a} F$ is the least $r$ such that $I^{r+1} F=2 I^{r} F$. The field $F$ satisfies $A_{m}$ when there are no nonzero torsion $m$-fold Pfister forms over $F$.) As remarked in [ELTW, p. 1165], when $I^{n+1} F$ is torsion-free, $s t_{r} F \leq n$ if and only if $s t_{a} F \leq n$. Hence $(\alpha) \Rightarrow(\beta)$. On the other hand, 
$s t_{r} F \leq s t_{a} F$ and for $m=2,3$ property $A_{m}$ implies $I^{m} F$ is torsion-free, by $\left[\mathrm{EL}_{1}\right.$, Theorem 2.8] and [EL 2 , Theorem 3]. So $(\beta) \Rightarrow(\alpha)$.

It is shown in [Le, Proposition 3.8] that the properties $(\beta)$ are inherited by quadratic extensions. (This is proved in [EP, Theorem 3.3] for the extension $F(\sqrt{-1}) / F$.) Hence, the properties $(\alpha)$ for $n=1$ or $n=2$, which we have assumed for $F$, also hold for every 2-extension of $F$. With this information, the proofs of Theorems 1.11 and 1.13 carry through unchanged.

Remark. To cut down on the hereditary hypotheses appearing in condition $(*)$, for $n \geq 2$ we could replace $(*)$ by the following condition: Assume $I^{n+1} K$ is torsion free for all finite degree extensions $K$ of $F$, and $s t_{r} F \leq n-1$. For, from this hypothesis we recover $(*)$ by the theorem of Bröcker [Br, Satz 4.3] which says $s t_{r} K \leq s t_{r} F+1$ whenever $K$ is algebraic over $F$.

\section{The TRANSFER IDEAL OF A MULTIQUADRATIC EXTENSION}

In this section we specialize to the case of multiquadratic extensions $K / F$. We give an inductive approach which will allow us to compute $\mathscr{T}_{K / F}$ for $K$ a multiquadratic extension whenever $F$ is a local field ( $\S 3)$, a global field ( $\S 4)$, or a field with $\tilde{u}(F) \leq 2(\S 5)$.

For any field $F$ and any $a \in F^{*}$, set $N_{F}(a)=\operatorname{im}\left(N_{F(\sqrt{a}) / F}\right) \subseteq F^{*} / F^{* 2}$. So $N_{F}(a)=D_{F}\langle\langle-a\rangle\rangle / F^{* 2}$.

The following notation will be used for Lemmas 2.1-2.3: Let $F$ be a field, $K=F\left(\sqrt{b_{0}}, \sqrt{b_{1}}, \ldots, \sqrt{b_{n}}\right)$ for some $b_{0}, \ldots, b_{n} \in F^{*}$, and $L=F\left(\sqrt{b_{0}}\right)$.

Lemma 2.1. The following statements are equivalent:

(1) For any $c_{1}, \ldots, c_{n} \in F^{*}$, if $\bigcap_{i=1}^{n} D_{L}\left(c_{i}\left\langle\left\langle-b_{i}\right\rangle\right\rangle\right) \neq \varnothing$, then $\bigcap_{i=1}^{n} D_{L}\left(c_{i}\left\langle\left\langle-b_{i}\right\rangle\right)\right) \cap F^{*} \neq \varnothing$.

(2) $N_{L / F}\left(\bigcap_{i=1}^{n} N_{L}\left(b_{i}\right)\right)=\bigcap_{i=0}^{n} N_{F}\left(b_{i}\right)$.

Proof. If $L=F$, then $b_{0} \in F^{* 2}$, so $N_{F}\left(b_{0}\right)=F^{*} / F^{* 2}$ and (1) and (2) hold trivially. Thus, we may assume $[L: F]=2$. Let $s_{*}^{L / F}$ denote the transfer map induced by the $F$-linear map $s^{L / F}: L \rightarrow F$ given by $s^{L / F}(1)=0$ and $s^{L / F}\left(\sqrt{b_{0}}\right)=1$.

$(2) \Rightarrow(1)$ Let $l \in \bigcap_{i=1}^{n} D_{L}\left(c_{i}\left\langle\left\langle-b_{i}\right\rangle\right\rangle\right)$, and let $t=N_{L / F}(l)$. We have $l\left\langle\left\langle-b_{i}\right\rangle\right\rangle \cong_{L} c_{i}\left\langle\left\langle-b_{i}\right\rangle\right\rangle, 1 \leq i \leq n$. Since $l\left\langle\left\langle-b_{i}\right\rangle\right\rangle$ is defined over $F, 0=$ $s_{*}^{L / F}\left(l\left\langle\left\langle-b_{i}\right\rangle\right\rangle\right)=r\left\langle\left\langle-t,-b_{i}\right\rangle\right\rangle$ for some $r \in F^{*}$. Therefore, $t \in \bigcap_{i=0}^{n} N_{F}\left(b_{i}\right)$. From (2) we conclude there exists $m \in \bigcap_{i=1}^{n} N_{L}\left(b_{i}\right)$ such that $N_{L / F}(m)=t=$ $N_{L / F}(l)$. Since the sequence $F^{*} / F^{* 2} \rightarrow L^{*} / L^{* 2} \stackrel{N_{L / F}}{\longrightarrow} F^{*} / F^{* 2}$ is exact (cf. [L, p. 202]) there is a $d \in F^{*}$ with $d m / l \in L^{* 2}$. Thus, for $1 \leq i \leq n, d\left\langle\left\langle-b_{i}\right\rangle\right\rangle \cong_{L}$ $d m\left\langle\left\langle-b_{i}\right\rangle\right\rangle \cong_{L} l\left\langle\left\langle-b_{i}\right\rangle\right\rangle \cong_{L} c_{i}\left\langle\left\langle-b_{i}\right\rangle\right\rangle$. This shows $d \in \bigcap_{i=1}^{n} D_{L}\left(c_{i}\left\langle\left\langle-b_{i}\right\rangle\right\rangle\right) \cap F^{*}$, proving (1).

(1) $\Rightarrow(2)$ The inclusion $\subseteq$ in (2) is clear. Let $t \in \bigcap_{i=0}^{n} N_{F}\left(b_{i}\right)$, and take any $l \in L^{*}$ with $N_{L / F}(l)=t$. Then $s_{*}^{L / F}\left(l\left\langle\left\langle-b_{i}\right\rangle\right\rangle\right)=r\left\langle\left\langle-t,-b_{i}\right\rangle\right\rangle=0$ since 
$t \in N_{F}\left(b_{i}\right)$. Therefore $l\left\langle\left\langle-b_{i}\right\rangle\right\rangle \cong{ }_{L} c_{i}\left\langle\left\langle-b_{i}\right\rangle\right\rangle$ for some $c_{i} \in F^{*}$. Since $l \in$ $\bigcap_{i=1}^{n} D_{L}\left(c_{i}\left\langle\left\langle-b_{i}\right\rangle\right\rangle\right),(1)$ implies there exists $d \in \bigcap_{i=1}^{n} D_{L}\left(c_{i}\left\langle\left\langle-b_{i}\right\rangle\right\rangle\right) \cap F^{*}$. Let $m=d l$. Then $m=d l \in \bigcap_{i=1}^{n} D_{L}\left(l c_{i}\left\langle\left\langle-b_{i}\right\rangle\right\rangle\right)=\bigcap_{i=1}^{n} D_{L}\left\langle\left\langle-b_{i}\right\rangle\right\rangle$. This shows $m \in \bigcap_{i=1}^{n} N_{L}\left(b_{i}\right)$ and $N_{L / F}(m)=d^{2} t \equiv t\left(\bmod F^{* 2}\right) ;$ hence $\bigcap_{i=0}^{n} N_{F}\left(b_{i}\right) \subseteq$ $N_{L / F}\left(\bigcap_{i=1}^{n} N_{L}\left(b_{i}\right)\right)$ proving (2).

Remark. Condition (2) of Lemma 2.1 does not depend on the choice of generators of $F\left(\sqrt{b_{1}}, \ldots, \sqrt{b_{n}}\right)$ over $F$, as one can see using the fact that $N_{F}(b) \cap N_{F}(c)=N_{F}(b) \cap N_{F}(c) \cap N_{F}(b c)$. (For this, note that $x \in N_{F}(b) \cap$ $N_{F}(c) \Rightarrow b, c \in N_{F}(x) \Rightarrow b c \in N_{F}(x) \Rightarrow x \in N_{F}(b c)$.) Thus, condition (1) does not depend on the choice of the generators either.

Lemma 2.2. Suppose the statements in Lemma 2.1 hold for $K / F^{\prime}$ for all fields $F^{\prime}$ with $F \subseteq F^{\prime} \subseteq K$. Then $G_{K / F}=\bigcap_{i=0}^{n} N_{F}\left(b_{i}\right)$.

Proof. The inclusion $\subseteq$ is clear, and the other inclusion follows by an easy induction using (2) of Lemma 2.1.

Lemma 2.3. If $G_{K / F}=\bigcap_{i=0}^{n} N_{F}\left(b_{i}\right)$ and for $m=1$ or $2, I^{m+1} F$ is torsion-free and $s t_{r} F \leq m$, then

$$
\mathscr{T}_{K / F}=\bigcap_{i=0}^{n} \operatorname{ann}\left\langle\left\langle-b_{i}\right\rangle\right\rangle=\bigcap_{i=0}^{n} \mathscr{T}_{F\left(\sqrt{b_{i}}\right) / F} .
$$

Proof. The second equality follows from Proposition 1.8. In the first equality the inclusion $\subseteq$ holds as $\mathscr{T}_{K / F} \subseteq \mathscr{T}_{F\left(\sqrt{b_{i}}\right) / F}=$ ann $\left\langle\left\langle-b_{i}\right\rangle\right\rangle$. For the other inclusion, take $q \in \bigcap_{i=0}^{n} \mathscr{T}_{F\left(\sqrt{b_{i}}\right) / F}$. Then by Lemma 1.7(1), $d_{ \pm}(q) \in \bigcap_{i=0}^{n} G_{F\left(\sqrt{b_{i}}\right) / F}$ $=\bigcap_{i=0}^{n} N_{F}\left(b_{i}\right)=G_{K / F}$. Suppose $P$ is an ordering of $F$ which does not extend to $K$. Then some $b_{i}<_{P} 0$. Hence $\operatorname{sgn}_{P}(q)=0$ since $q \in \operatorname{ann}\left\langle\left\langle-b_{i}\right\rangle\right\rangle$. Corollary 1.14 implies $q \in \mathscr{T}_{K / F}$.

In [STW] a property $P_{1}(3)$ was considered. It was shown [STW, Proposition 2.8] that a field $F$ satisfies property $P_{1}(3)$ if and only if condition (1) of Lemma 2.1 holds for all $b_{0}, b_{1}, b_{2} \in F^{*}$. It was also shown in [STW, §5] that if $F$ is finitely generated of positive transcendence degree over $\mathbb{Q}$ or is finitely generated of transcendence degree 2 or more over any field of characteristic 0 , then $F$ does not satisfy $P_{1}(3)$. These examples yield counterexamples to the first question of the introduction.

Proposition 2.4. Let $F$ be a field not satisfying property $P_{1}(3)$. Then there exist $b_{0}, b_{1}, b_{2} \in F^{*}$ such that for $K=F\left(\sqrt{b_{0}}, \sqrt{b_{1}}, \sqrt{b_{2}}\right)$,

$$
G_{K / F} \varsubsetneqq \bigcap_{i=0}^{n} N_{F}\left(b_{i}\right) \text { and } \mathscr{T}_{K / F} \varsubsetneqq \bigcap_{i=0}^{n} \mathscr{T}_{F\left(\sqrt{b_{i}}\right) / F} .
$$


Proof. Since $F$ does not satisfy $P_{1}(3)$ there are $b_{0}, b_{1}, b_{2} \in F^{*}$ for which (1) of Lemma 2.1 fails to hold. Use these $b_{i}$. Then, with $L=F\left(\sqrt{b_{0}}\right)$ we have

$$
G_{K / F} \subseteq N_{L / F}\left(N_{L}\left(b_{1}\right) \cap N_{L}\left(b_{2}\right)\right) \varsubsetneqq \bigcap_{i=0}^{2} N_{F}\left(b_{i}\right),
$$

since (2) of Lemma 2.1 must also fail. So, for any $c \in \bigcap_{i=0}^{2} N_{F}\left(b_{i}\right)$ with $c \notin G_{K / F}, \quad\langle\langle-c\rangle\rangle \in \bigcap_{i=0}^{2} \mathscr{T}_{F\left(\sqrt{b_{i}}\right) / F}$, but $\langle\langle-c\rangle\rangle \notin \mathscr{T}_{K / F}$ by Lemma 1.7.

\section{THE TRANSFER IDEAL FOR LOCAL FIELDS}

We now calculate $\mathscr{T}_{K / F}$ and the related quantities defined in $\S 1$ for $F \subseteq K$ local fields, i.e., fields with complete discrete valuation and finite residue field.

Theorem 3.1. Let $F$ be a local field and $K=F\left(\sqrt{b_{0}}, \ldots, \sqrt{b_{n}}\right)$ where $b_{0}, \ldots$, $b_{n} \in F^{*}$. Then,

(1) $G_{K / F}=\bigcap_{i=0}^{n} N_{F}\left(b_{i}\right)$.

(2) $\mathscr{T}_{K / F}=\bigcap_{i=0}^{n}$ ann $\left\langle\left\langle-b_{i}\right\rangle\right\rangle=\bigcap_{i=0}^{n} \mathscr{T}_{F\left(\sqrt{b_{i}}\right) / F}$.

Proof. Let $L=F\left(\sqrt{b_{0}}\right)$. Recall that if $[L: F]=2$ then every three-dimensional quadratic form defined over $F$ becomes isotropic over $L$ (see [L, p. 158, Corollary 2.15]). Statement (1) of Lemma 2.1 follows easily when $[L: F]=$ 2 , and holds trivially when $[L: F]=1$. Hence, Lemma 2.2 shows $G_{K / F}=$ $\bigcap_{i=0}^{n} N\left(b_{i}\right)$, proving (1). Then Lemma 2.3 with $m=2$ yields (2), since $I^{3} F=$ 0 and st $_{r} F=0$.

Theorem 3.2. Let $K / F$ be an arbitrary finite degree extension of local fields and let $E$ be the maximal multiquadratic extension of $F$ contained in $K$. Then,

(1) $G_{K / F}=G_{E / F}$ and $\mathfrak{A}\left(G_{K / F}\right)=\mathfrak{A}\left(G_{E / F}\right)$.

(2) $\mathscr{T}_{K / F}=\mathscr{T}_{E / F}=$ ann $W(E / F)=$ ann $W(K / F)$, unless $[K: F]$ is even and $E=F$. In that case, $\mathscr{T}_{K / F}=$ ann $W(K / F)=I F$.

(3) $\mathfrak{A}\left(G_{K / F}\right)=\mathscr{T}_{K / F}$ if and only if $[K: F]$ is even and $[E: F]<\left|F^{*} / F^{* 2}\right|$.

Proof. Let $K^{\prime}$ be the maximal abelian Galois extension of $F$ in $K$, and let $L=F\left(\left\{\sqrt{a} \mid a \in F^{*}\right\}\right)$. Then $[L: F]=\left|F^{*}\right| F^{* 2} \mid<\infty$ and $E=K^{\prime} \cap L$. We have $G_{K / F}=G_{K^{\prime} / F}$ by [Se, p. 172, Proposition 3; p. 195, Theorem 1] and $G_{K^{\prime} / F} \cdot G_{L / F}=G_{E / F}$ by [Se, pp. 172-173, Proposition 4]. Since $F^{*} \bmod$ the group of norms from $L^{*}$ is isomorphic to the 2-torsion Galois group $\operatorname{Gal}(L / F)$ by [Se, p. 169], this norm group contains $F^{* 2}$. It follows from [Se, p. 172, Corollary] and Kummer theory that $\left|F^{*} / F^{* 2}: G_{L / F}\right|=|\mathrm{Gal}(L / F)|=\left|F^{*} / F^{* 2}\right|$. Therefore, $\left|G_{L / F}\right|=1$. In all, we now have $G_{K / F}=G_{K^{\prime} / F}=G_{K^{\prime} / F} \cdot G_{L / F}=$ 
$G_{E / F}$, proving (1). This shows, by Lemma $1.7(2)$, that $\mathscr{T}_{K / F}$ and $\mathscr{T}_{E / F}$ contain the same binary forms. In addition, $I^{2} F \subseteq \mathscr{T}_{K / F} \subseteq \mathscr{T}_{E / F}$ by Theorem 1.11. Assume $E \supsetneqq F$. Then, $\mathscr{T}_{K / F}=\mathscr{T}_{E / F}$ (see Lemma 1.7(4)). Let $E=F\left(\sqrt{a_{1}}, \ldots, \sqrt{a_{n}}\right)$. Since $W(E / F) \subseteq W(K / F)$ we have by Theorem 3.1(2) and Proposition 1.4,

$$
\begin{aligned}
\operatorname{ann} W(K / F) & \subseteq \operatorname{ann} W(E / F) \subseteq \bigcap_{i=1}^{n} \operatorname{ann}\left\langle\left\langle-a_{i}\right\rangle\right\rangle \\
& =\mathscr{T}_{E / F}=\mathscr{T}_{K / F} \subseteq \text { ann } W(K / F) .
\end{aligned}
$$

This proves the first sentence of (2), unless $[K: F]$ is odd. But the case $[K: F]$ odd is settled by Lemma 1.2. Now assume $[K: F]$ is even and $E=F$. By Lemma 1.7(4) we have $\mathscr{T}_{K / F}=I F$ since $G_{K / F}=G_{E / F}=F^{*} / F^{* 2}$. Thus, $I F=\mathscr{T}_{K / F} \subseteq$ ann $W(K / F) \subseteq I F$ by Lemma 1.4 and the fact that $W(K / F)$ contains the anisotropic 2-fold Pfister form. This completes the proof of (2).

For (3), we may assume $[K: F]$ is even (since otherwise Lemma 1.2 shows $\left.\mathfrak{A}\left(G_{K / F}\right)=I F \varsubsetneqq \mathscr{T}_{K / F}=W F\right)$. Then $\mathscr{T}_{K / F} \subseteq I F$. Now, $\mathfrak{A}\left(G_{K / F}\right)$ and $\mathscr{T}_{K / F}$ contain the same binary forms, and $\mathscr{T}_{K / F}$ contains the anisotropic 2-fold Pfister form, call it $\varphi$. Since $\varphi$ is a multiple of every anisotropic 2-dimensional form, $\mathscr{T}_{K / F}=\mathfrak{A}\left(G_{K / F}\right)$ if and only if $\mathfrak{A}\left(G_{K / F}\right) \neq 0$, if and only if $\left|G_{K / F}\right|>1$, if and only if $\left|G_{E / F}\right|>1$. By the same argument as above for $L$, the group of norms from $E$ to $F$ contains $F^{* 2}$, so that $\left|F^{*} / F^{* 2}: G_{E / F}\right|=|\operatorname{Gal}(E / F)|=[E: F]$. Therefore, $\left|G_{E / F}\right|>1$ if and only if $[E: F]<\left|F^{*} / F^{* 2}\right|$, proving (3).

\section{THE TRANSFER IDEAL FOR GLOBAL FIELDS, AND THE HASSE NORM THEOREM MOD SQUARES}

We begin this section by proving statement (1) of Lemma 2.1 for global fields. This will lead to a determination of $\mathscr{T}_{K / F}$ for $K$ a multiquadratic extension of a global field $F$, and to a Hasse norm theorem mod squares for such fields. Let $\Omega(F)$ denote the set of prime spots of a global field $F$. If $\mathfrak{p} \in \Omega(F)$ let $F_{\mathrm{p}}$ denote the $\mathfrak{p}$-adic completion of $F$ and, if $\mathfrak{p}$ is finite, let $v_{\mathfrak{p}}: F^{*} \rightarrow \mathbb{Z}$ be the (surjective) valuation of $F$ associated to $\mathfrak{p}$.

Theorem 4.1. Let $F$ be a global field, and let $a, b_{1}, \ldots, b_{n}, c_{1}, \ldots, c_{n} \in F^{*}$. Set $L=F(\sqrt{a})$. If $\bigcap_{i=1}^{n} D_{L}\left(c_{i}\left\langle\left\langle-b_{i}\right\rangle\right)\right) \neq \varnothing$, then $\bigcap_{i=1}^{n} D_{L}\left(c_{i}\left\langle\left\langle-b_{i}\right\rangle\right\rangle\right) \cap F^{*} \neq \varnothing$. Proof. We may assume $[L: F]=2$, and we may assume $a, b_{1}, \ldots, b_{n}$ are $\mathbb{Z}_{2}$-independent in $F^{*} / F^{* 2}$ since the remark after Lemma 2.1 shows we can use any set of generators of $F\left(\sqrt{b_{1}}, \ldots, \sqrt{b_{n}}\right)$ to prove the theorem. Let $\gamma_{i}=c_{i}\left\langle\left\langle-b_{i}\right\rangle\right\rangle$.

Assume there is an $l \in \bigcap_{i=1}^{n} D_{L}\left(\gamma_{i}\right)$. Let $T_{0}$ be a finite subset of $\Omega(F)$ containing all infinite primes, all dyadic primes, and all finite primes $\mathfrak{p}$ at which any of $v_{\mathfrak{p}}(a), v_{\mathfrak{p}}\left(b_{1}\right), \ldots, v_{\mathfrak{p}}\left(b_{n}\right), v_{\mathfrak{p}}\left(c_{1}\right), \ldots, v_{\mathfrak{p}}\left(c_{n}\right)$ is nonzero. Let $T_{1}$ be 
another finite subset of $\Omega(F)$, disjoint from $T_{0}$, such that, for each subset $\mathscr{S}$ of $\{1,2, \ldots, n\}$ there is a $\mathfrak{p} \in T_{1}$ such that $a \notin F_{\mathfrak{p}}^{* 2}$ and, for $1 \leq i \leq n$, $b_{i} \notin F_{\mathrm{p}}^{* 2}$ if and only if $i \in \mathscr{S}$. The existence of $T_{1}$ follows from the $\mathbb{Z}_{2}$ independence of $a, b_{1}, \ldots, b_{n}$, using the Global Square Theorem (cf. [OM, 65:17]). Let $T=T_{0} \cup T_{1}$.

For each $\mathfrak{p} \in T$, property (1) of Lemma 2.1 holds for the local field $F_{\mathfrak{p}}$ by the proof of Theorem 3.1 if $\mathfrak{p}$ is finite, and it holds trivially if $\mathfrak{p}$ is infinite. So there is an $r_{\mathfrak{p}} \in F_{\mathfrak{p}}^{*}$ satisfying $r_{\mathfrak{p}} \in \bigcap_{i=1}^{n} D_{L_{\mathfrak{p}}}\left(\gamma_{i}\right)$ for each $\mathfrak{P} \in \Omega(L), \mathfrak{P} \mid \mathfrak{p}$. (This argument applies if there is only one $\mathfrak{P} \mid \mathfrak{p}$. But if there are $\mathfrak{P}_{1} \neq \mathfrak{P}_{2} \in \Omega(L)$ with each $\mathfrak{P}_{i} \mid \mathfrak{p}$, then each $L_{\mathfrak{p}_{i}}=F_{\mathfrak{p}}$, and we can take $r_{\mathfrak{p}}=l_{\mathfrak{P}_{1}}$. )

Now, we adjust parities. Let

$$
\mathscr{S}=\left\{i \mid r_{\mathfrak{p}} \notin D_{F_{\mathfrak{p}}}\left(\gamma_{i}\right) \text { for an odd number of } \mathfrak{p} \in T\right\} .
$$

Choose a $\mathfrak{p}_{0} \in T_{1}$ such that $a \notin F_{\mathfrak{p}_{0}}^{* 2}$ and, for $1 \leq i \leq n, \quad b_{i} \notin F_{\mathfrak{p}_{0}}^{* 2}$ if and only if $i \in \mathscr{S}$. If $\pi_{0}$ is any uniformizer for $v_{p_{0}}$, let $t_{\mathfrak{p}_{0}}=\pi_{0} r_{p_{0}}$. Take any $i \in \mathscr{S}$. Then $\gamma_{i F_{\mathrm{p}_{0}}}$ is an anisotropic unit form, so $t_{\mathrm{p}_{0}} \in D_{F_{\mathrm{p}_{0}}}\left(\gamma_{i}\right)$ if and only if $r_{\mathfrak{p}_{0}} \notin D_{F_{p_{0}}}\left(\gamma_{i}\right)$. Nonetheless, for $\mathfrak{P}_{0} \in \Omega(L)$ with $\mathfrak{P}_{0} \mid \mathfrak{p}_{0}$, we have $t_{\mathfrak{p}_{0}} \in D_{L_{\mathfrak{p}_{0}}}\left(\gamma_{i}\right)$ as $b_{i} \in L_{\mathfrak{P}_{0}}^{* 2}\left(\right.$ since $\left.b_{i} \equiv a\left(\bmod F_{\mathfrak{p}_{0}}^{* 2}\right)\right)$. On the other hand, if $i \notin \mathscr{S}$ then $\gamma_{i F_{p_{0}}}$ is isotropic, so $r_{p_{0}}, t_{p_{0}} \in D_{F_{p_{0}}}\left(\gamma_{i}\right)$. Set $t_{p}=r_{p}$ for all $\mathfrak{p} \in T-\left\{\mathfrak{p}_{0}\right\}$. By this adjustment at $\mathfrak{p}_{0}$ we have assured that for each $i$, $1 \leq i \leq n,\left\{\mathfrak{p} \in T \mid t_{\mathfrak{p}} \notin D_{F_{\mathfrak{p}}}\left(\gamma_{i}\right)\right\}$ is even while $t_{\mathfrak{p}} \in D_{L_{\mathfrak{p}}}\left(\gamma_{i}\right)$ for each $\mathfrak{p} \in T$ and $\mathfrak{P} \mid \mathfrak{p}$.

By the weak approximation theorem there is a $t \in F^{*}$ such that $t \equiv t_{\mathrm{p}}$ $\left(\bmod F_{\mathfrak{p}}^{* 2}\right)$ for each $\mathfrak{p} \in T$. Let $R=\left\{\mathfrak{p}_{1}, \ldots, \mathfrak{p}_{k}\right\}$ be the set of primes $\mathfrak{p}$ of $\Omega(F)-T$ at which $v_{\mathfrak{p}}(t)$ is odd. Let $\mathfrak{M}=\prod_{\mathfrak{p} \in T} \mathfrak{p}^{e(\mathfrak{p})}$ be a modulus (cf. [J, p. 107]) with exponents $e(\mathfrak{p})$ chosen sufficiently large that $F_{\mathfrak{M}, 1} \subseteq F_{\mathfrak{p}}^{* 2}$ for each $\mathfrak{p} \in T$. (Here,

$$
F_{\mathfrak{M}, 1}=\left\{c \in F^{*} \mid v_{\mathfrak{p}}(c)=0 \text { for each finite } \mathfrak{p} \in T \text { and } c \equiv 1(\bmod \mathfrak{M})\right\},
$$

cf. [J, p. 108].) Let $I^{\mathfrak{M}}$ be the part of the ideal group of $F$ generated by $\Omega(F)-T$, and let $i\left(F_{\mathfrak{M}, 1}\right)$ be the image of $F_{\mathfrak{M}, 1}$ in $I^{\mathfrak{M}}$. By the Frobenius density theorem [J, p. 137, Theorem 5.8] or [W, p. 290, Corollary] there is a prime $\mathfrak{q} \in \Omega(F)-(T \cup R)$ lying in the coset of $\left(\mathfrak{p}_{1} \ldots \mathfrak{p}_{k}\right)^{-1}$ in the finite ray class group $I^{\mathfrak{M}} / i\left(F_{\mathfrak{M}, 1}\right)$. That is, there is an $x \in F_{\mathfrak{M}, 1}$ such that $(x)=\left(\mathfrak{p}_{1}\right) \cdots\left(\mathfrak{p}_{k}\right)(\mathfrak{q})$ in the ring of integers of $F$.

We claim that $t x \in \bigcap_{i=1}^{n} D_{L}\left(\gamma_{i}\right) \cap F^{*}$. This will complete the proof. It suffices by the Hasse-Minkowski Theorem [OM, 66:4] to verify that $t x \in D_{L_{\mathfrak{P}}}\left(\gamma_{i}\right)$ for each $\mathfrak{P} \in \Omega(L)$ and each $i$. Take any $\mathfrak{P} \in \Omega(L)$ and let $\mathfrak{p}$ be the restriction of $\mathfrak{P}$ to $\Omega(F)$. If $\mathfrak{p} \in T$, then $x \in F_{\mathfrak{p}}^{* 2} ;$ so, $\bmod L_{\mathfrak{p}}^{* 2}, t x \equiv t \equiv t_{\mathfrak{p}} \in D_{L_{\mathfrak{p}}}\left(\gamma_{i}\right)$ for each $i$. If $\mathfrak{p} \in \Omega(F)-(T \cup\{\mathfrak{q}\})$, then $\gamma_{i}$ is a unit form and $v_{\mathfrak{p}}(t x)$ is even, so 
$t x \in D_{F_{\mathfrak{p}}}\left(\gamma_{i}\right) \subseteq D_{L_{\mathfrak{p}}}\left(\gamma_{i}\right)$. There remains only $\mathfrak{p}=\mathfrak{q}$. But note that by Hilbert's reciprocity law [S, p. 221, Theorem 5.1] (applied to $\langle\langle-t x\rangle\rangle \otimes \gamma_{i}$ ) for each $i$ the set $S_{i}=\left\{\mathfrak{r} \in \Omega(F) \mid t x \notin D_{F_{\mathrm{r}}}\left(\gamma_{i}\right)\right\}$ is finite of even cardinality. Also, we have just shown that $S_{i} \subseteq T \cup\{\mathfrak{q}\}$. But for $\mathfrak{r} \in T, x t \equiv t_{\mathfrak{r}}\left(\bmod F_{\mathfrak{r}}^{* 2}\right)$, so $\left|S_{i} \cap T\right|$ is even by the earlier parity adjustment. Hence, $\mathfrak{q} \notin S_{i}$ for each $i$, i.e., $t x \in D_{F_{\mathfrak{q}}}\left(\gamma_{i}\right) \subseteq D_{L_{\mathfrak{Q}}}\left(\gamma_{i}\right)$ for each $\mathfrak{Q} \mid \mathfrak{q}$. This proves the claim.

Theorem 4.2. Let $F$ be a global field, and $K=F\left(\sqrt{a_{1}}, \ldots, \sqrt{a_{n}}\right)$, for any $a_{1}, \ldots, a_{n} \in F^{*}$. Then,

(1) $G_{K / F}=\bigcap_{i=1}^{n} N_{F}\left(a_{i}\right)$.

(2) $\mathscr{T}_{K / F}=\bigcap_{i=1}^{n}$ ann $\left\langle\left\langle-a_{i}\right\rangle\right\rangle=\bigcap_{i=1}^{n} \mathscr{T}_{F\left(\sqrt{a_{i}}\right) / F}$.

Proof. (1) follows from Theorem 4.1 and Lemma 2.2. Recall (e.g. from [L, p. 172, Corollary 3.9]) that for any global field $E, I^{3} E$ is torsion-free and $s_{r} E \leq 1$ as $E$ satisfies SAP. Hence, $F$ satisfies (*) with $n=2$, so (2) follows from (1) and Lemma 2.3.

If $c \in F^{*}$ and $\mathfrak{p} \in \Omega(F)$, we let $c_{\mathfrak{p}}$ denote the image of $c$ under the embedding $F \rightarrow F_{\mathfrak{p}}$.

Definition 4.3. Let $E / F$ be a finite degree extension of global fields.

(1) Let $L=\left\{c \in F^{*} \mid c_{\mathfrak{p}} F_{\mathfrak{p}}^{* 2} \in G_{E_{\mathfrak{p}} / F_{\mathfrak{p}}}\right.$ for all $\left.\mathfrak{P} \in \Omega(E), \mathfrak{p} \in \Omega(F), \mathfrak{P} \mid \mathfrak{p}\right\}$, i.e., $L$ is the group of local norms mod squares. Let $G=\left\{c \in F^{*} \mid c F^{* 2}\right.$ $\left.\in G_{E / F}\right\}$, i.e., $G$ is the group of global norms mod squares. We say the Hasse norm theorem mod squares holds for $E / F$ if $L \subseteq G$.

(2) We say there is a local global theorem for $\mathscr{T}_{E / F}$ if for $q \in W F, q_{\mathfrak{p}} \in$ $\mathscr{T}_{E_{\mathfrak{p}} / F_{\mathfrak{p}}}$ for all $\mathfrak{p} \in \Omega(F), \mathfrak{P} \mid \mathfrak{p}$, implies $q \in \mathscr{T}_{E / F}$.

Theorem 4.4. Let $E / F$ be a finite degree extension of global fields. Then there is a local global theorem for $\mathscr{T}_{E / F}$ if and only if the Hasse norm theorem mod squares holds for $E / F$.

Proof. Suppose there is a local global theorem for $\mathscr{T}_{E / F}$. Let $c \in F^{*}$, and suppose $c_{\mathfrak{p}} F_{\mathfrak{p}}^{* 2} \in G_{E_{\mathfrak{p}} / F_{\mathfrak{p}}}$ for all $\mathfrak{p} \in \Omega(F), \mathfrak{P} \mid \mathfrak{p}$. Then $q=\langle 1,-c\rangle$ satisfies $q_{\mathfrak{p}} \in \mathscr{T}_{E_{\mathfrak{p}} / F_{\mathfrak{p}}}$ for all $\mathfrak{p} \in \Omega(F)$, and hence $q \in \mathscr{T}_{E / F}$ by the local global theorem. It follows that $c=d_{ \pm}(q) \in G_{E / F}$ by Lemma 1.7(1), so the Hasse norm theorem mod squares holds for $E / F$.

Now assume the Hasse norm theorem mod squares holds for $E / F$. Suppose $q \in W F$ with $q_{\mathfrak{p}} \in \mathscr{T}_{E_{\mathfrak{p}} / F_{\mathfrak{p}}}$ for all $\mathfrak{p} \in \Omega(F), \mathfrak{P} \mid \mathfrak{p}$. Then $d_{ \pm}\left(q_{\mathfrak{p}}\right) \in G_{E_{\mathfrak{p}} / F_{\mathfrak{p}}}$ for all $\mathfrak{p} \in \Omega(F)$, so $d_{ \pm}(q) \in G_{E / F}$ by the Hasse norm theorem mod squares. Let $\mathfrak{p}$ be an infinite prime of $F$ corresponding to an ordering $P$ of $F$ that does not extend to $E$. Then $\mathscr{T}_{E_{\mathfrak{p}} / F_{\mathrm{p}}}=\mathscr{T}_{\mathbf{C} / \mathbf{R}}=0$ since ann $\langle\langle 1\rangle\rangle=0$ in $W \mathbf{R}$. Therefore, $q_{\mathfrak{p}}=0$, which implies that $\operatorname{sgn}_{P}(q)=0$. Since a global field satisfies (*) with $n=2$, Theorem 1.13 implies $q \in \mathscr{T}_{E / F}$. 
Remark. If $E / F$ is a finite degree Galois extension of global fields, then every global norm in $F$ from $E$ is a local norm from $E$. In particular, $G \subseteq L$ (see Definition 4.3). Similarly, if $q \in \mathscr{T}_{E / F}$ then $q_{\mathfrak{p}} \in \mathscr{T}_{E_{\mathfrak{p}} / F_{\mathfrak{p}}}$ for all $\mathfrak{p} \in \Omega(F)$, $\mathfrak{P} \mid \mathfrak{p}$, by Lemma 1.5. But none of these assertions is true in general if $E / F$ is not Galois. (To see this it suffices to consider an example with $[E: F]=3$ and a $\mathfrak{p} \in \Omega(F)$ with exactly two $\left.\mathfrak{P}_{i} \in \Omega(E), \mathfrak{P}_{i} \mid \mathfrak{p}.\right)$

Theorem 4.5. Let $F$ be a global field and let $K=F\left(\sqrt{a_{1}}, \ldots, \sqrt{a_{n}}\right)$ for $a_{1}, \ldots$, $a_{n} \in F^{*}$. Then the Hasse norm theorem mod squares holds for $K / F$ and there is a local global theorem for $\mathscr{T}_{K / F}$.

Proof. Take $c \in F^{*}$ and suppose $c_{\mathfrak{p}} F_{\mathfrak{p}}^{* 2} \in G_{K_{\mathfrak{p}} / F_{\mathfrak{p}}}$ for all $\mathfrak{p} \in \Omega(F)$ and $\mathfrak{P} \in$ $\Omega(K)$ with $\mathfrak{P} \mid \mathfrak{p}$. Then $c_{\mathfrak{p}} F_{\mathfrak{p}}^{* 2} \in \bigcap_{i=1}^{n} N_{F_{\mathfrak{p}}}\left(a_{i}\right)$ for all $\mathfrak{p} \in \Omega(F)$, by Theorem 3.1 if $\mathfrak{p}$ is finite, and trivially if $\mathfrak{p}$ is infinite. By the usual Hasse Norm Theorem for quadratic extensions [OM, 65:23] it follows that $c F^{* 2} \in \bigcap_{i=1}^{n} N_{F}\left(a_{i}\right)=G_{K / F}$ by Theorem 4.2(1). This shows the Hasse norm theorem mod squares holds for $K / F$, and the local global theorem for $\mathscr{T}_{K / F}$ follows by Theorem 4.4.

Remark 4.6. Note that the extension $\mathbb{Q}(\sqrt{13}, \sqrt{17}) / \mathbb{Q}$ fails to satisfy the usual Hasse norm theorem (cf. [CF, p. 360]) but we have just shown that the Hasse norm theorem mod squares does hold. However, this seems to be rather special to multiquadratic extensions. In a forthcoming paper [LW] we will show that for any odd prime $q$ the Hasse norm theorem mod $q$ does not always hold for elementary abelian $q$-extensions of global fields. We will also show that the Hasse norm theorem mod squares does not always hold for Galois extensions with group $\mathbb{Z} / 2 \mathbb{Z} \times \mathbb{Z} / 2^{m} \mathbb{Z}$, for $m \geq 2$. This yields examples of extensions for which the local global theorem for the transfer ideal fails to hold.

\section{THE TRANSFER IDEAL FOR FIELDS WITH $\tilde{u}(F) \leq 2$}

We turn now to another class of fields for which we can compute the transfer ideal. Let $F$ be a field with Hasse number $\tilde{u}(F) \leq 2$. This means that every three-dimensional quadratic form over $F$ which is indefinite with respect to every ordering of $F$ is isotropic. Examples of such fields are algebraic extensions of $R(x)$ where $R$ is a real closed field or even a hereditarily Euclidean field. (See, e.g., [E, Ex. 4.9], [ELP, Theorem I].)

Theorem 5.1. Let $F$ be a field with $\tilde{u}(F) \leq 2$. Then the statements in Lemma 2.1 are true for any $b_{0}, \ldots, b_{n} \in F^{*}$.

Proof. We prove (1) in Lemma 2.1. Let $L=F\left(\sqrt{b_{0}}\right)$. Recall that $\tilde{u}(L) \leq 2$ by [ELW ${ }_{4}$, Proposition 3.3], since $\tilde{u}(F) \leq 2$ and $[L: F] \leq 2$. For $a \in L^{*}$, let $H_{L}(a)=\left\{P \in X_{L} \mid a>_{P} 0\right\}$, where $X_{L}$ is the space of orderings of $L$. For any $b_{1}, \ldots, b_{n}, c_{1}, \ldots, c_{n} \in F^{*}$, let

$$
E_{1}=\bigcup_{i=1}^{n}\left(H_{L}\left(-b_{i}\right) \cap H_{L}\left(c_{i}\right)\right) \quad \text { and } \quad E_{2}=\bigcup_{i=1}^{n}\left(H_{L}\left(-b_{i}\right) \cap H_{L}\left(-c_{i}\right)\right) \text {. }
$$


We claim that

$$
\bigcap_{i=1}^{n} D_{L}\left(c_{i}\left\langle\left\langle-b_{i}\right\rangle\right\rangle\right) \neq \varnothing \quad \text { if and only if } \quad E_{1} \cap E_{2}=\varnothing
$$

For, if $E_{1} \cap E_{2} \neq \varnothing$ there is a $P \in X_{L}$ and there are indices $i, j$, such that $b_{i}<_{P} 0, b_{j}<_{P} 0$, and $c_{i} c_{j}<_{P} 0$. Then one of $c_{i}\left\langle\left\langle-b_{i}\right\rangle\right\rangle, c_{j}\left\langle\left\langle-b_{j}\right\rangle\right\rangle$ is positive definite and the other is negative definite; so $D_{L}\left(c_{i}\left\langle\left\langle-b_{i}\right\rangle\right\rangle\right) \cap D_{L}\left(c_{j}\left\langle\left\langle-b_{j}\right\rangle\right\rangle\right)=\varnothing$. On the other hand, if $E_{1} \cap E_{2}=\varnothing$ there is an $l \in L^{*}$ with $l>_{P} 0$ for $P \in E_{1}$ and $l<_{P} 0$ for $P \in E_{2}$. (This uses the SAP property for $L$, which holds as $\tilde{u}(L) \leq 2$, cf. [ELP, Theorem F].) Then for each $i,\left\langle c_{i},-b_{i} c_{i},-l\right\rangle$ is totally indefinite, hence isotropic as $\tilde{u}(L) \leq 2$. Thus, $l \in \bigcap_{i=1}^{n} D_{L}\left(c_{i}\left\langle\left\langle-b_{i}\right\rangle\right\rangle\right)$, as claimed.

Now, suppose $\bigcap_{i=1}^{n} D_{L}\left(c_{i}\left\langle\left\langle-b_{i}\right\rangle\right\rangle\right) \neq \varnothing$. Then $E_{1} \cap E_{2}=\varnothing$. Hence, because $E_{1}$ and $E_{2}$ are defined by elements of $F, \varepsilon_{L / F}\left(E_{1}\right) \cap \varepsilon_{L / F}\left(E_{2}\right)=\varnothing$. Using SAP for $F$, which holds as $\tilde{u}(F) \leq 2$, we find an $f \in F^{*}$ with $f>_{P} 0$ if $P \in \varepsilon_{L / F}\left(E_{1}\right)$ and $f<_{P} 0$ if $P \in \varepsilon_{L / F}\left(E_{2}\right)$. Then $f \in \bigcap_{i=1}^{n} D_{L}\left(c_{i}\left\langle\left\langle-b_{i}\right\rangle\right\rangle\right) \cap F^{*}$ by the same argument as for $l$ above. This proves (1) of Lemma 2.1 .

Corollary 5.2. Let $F$ be a field with $\tilde{u}(F) \leq 2$ and let $K=F\left(\sqrt{b_{0}}, \ldots, \sqrt{b_{n}}\right)$, for any $b_{0}, \ldots, b_{n} \in F^{*}$. Then,

(1) $G_{K / F}=\bigcap_{i=0}^{n} N_{F}\left(b_{i}\right)$.

(2) $\mathscr{T}_{K / F}=\bigcap_{i=0}^{n}$ ann $\left\langle\left\langle-b_{i}\right\rangle\right\rangle=\bigcap_{i=0}^{n} \mathscr{T}_{F\left(\sqrt{b_{i}}\right) / F}$.

Proof. Every multiquadratic extension $E$ of $F$ satisfies $\tilde{u}(E) \leq 2$ by $\left[E L W_{4}\right.$, Proposition 3.3]. Therefore, Theorem 5.1 and Lemma 2.2 imply statement (1). Since $\tilde{u}(F) \leq 2, I^{2} F$ is torsion free and $F$ is a SAP field, i.e., $s t_{r} F \leq 1$. So statement (2) follows by Lemma 2.3 .

We close by deducing from the results of $\S 1$ a kind of "local global principle for $\mathscr{G}_{K / F}$ " for fields which satisfy hereditarily $\tilde{u} \leq 2$.

\section{Corollary 5.3.}

(1) Assume $\tilde{u}(K) \leq 2$ for every finite degree extension $K$ of a field $F$. Then for $q \in W F, q \in \mathscr{T}_{K / F}$ if and only if $\operatorname{sgn}_{P}(q)=0$ for all orderings $P$ of $F$ which do not extend to $K$.

(2) If one assumes only that $\tilde{u}(F) \leq 2$, then the conclusion of (1) holds if $K$ is a 2-extension of $F$.

Proof. Condition (*) with $n=1$ is equivalent to $\tilde{u}(K) \leq 2$ for all finite degree extensions $K / F$, since $\tilde{u}(E) \leq 2$ if and only if $I^{2} E$ is torsion-free and $E$ is a SAP field (cf. [ELP, Theorem F]). Thus, Theorem 1.13 yields (1) and Corollary 1.14 gives $(2)$. 


\section{REFERENCES}

[A] J. Kr. Arason, Cohomologische Invarianten quadratischer Formen, J. Algebra, 36 (1975), 448491.

[Bo] R. Bos, Quadratic forms, orderings, and abstract Witt rings, Doctoral thesis, Univ. of Utrecht, The Netherlands, 1984.

[Br] L. Bröcker, Zur Theorie der quadratischen Formen über formal reellen Körpern, Math. Ann. 210 (1974), 233-256.

[CF] J. W. S. Cassels and A. Fröhlich, eds., Algebraic number theory, Academic Press, London, 1967.

[E] R. Elman, Quadratic forms and the u-invariant. III, Conference on Quadratic Forms-1976 (G. Orzech, ed.), Queen's Papers in Pure and Appl. Math., Vol. 46, Queen's University, Kingston, Ontario, 1977, pp. 422-444.

[EL $\left.\mathrm{E}_{1}\right]$ R. Elman and T.-Y. Lam, Quadratic forms over formally real fields and Pythagorean fields, Amer. J. Math. 94 (1972), 1155-1194.

$\left[\mathrm{EL}_{2}\right]$ _ Classification theorems for quadratic forms over fields, Comment. Math. Helv. 49 (1974), 373-381.

[ELP] R. Elman, T.-Y. Lam, and A. Prestel, On some Hasse principles over formally real fields, Math. Z. 134 (1973), 291-301.

[ELTW] R. Elman, T.-Y. Lam, J.-P. Tignol, and A. R. Wadsworth, Witt rings and Brauer groups under multiquadratic extensions. I, Amer. J. Math. 105 (1983), 1119-1170.

[ELW 1 ] R. Elman, T.-Y. Lam, and A. R. Wadsworth, Amenable fields and Pfister extensions, Conference on Quadratic Forms-1976 (G. Orzech, ed.), Queen's Papers in Pure and Appl. Math., Vol. 46, Queen's University, Kingston, Ontario, 1977, pp. 445-492.

$\left[\mathrm{ELW}_{2}\right]$ _ , Pfister ideals in Witt rings, Math. Ann. 245 (1979), 219-245.

$\left[\mathrm{ELW}_{3}\right]$ _ _ Orderings under field extensions, J. Reine Angew. Math. 306 (1979), 7-27.

$\left[\mathrm{ELW}_{4}\right]$ _ _ Quadratic forms under multiquadratic extensions, Indag. Math. 42 (1980), 131-145.

[EP] R. Elman and A. Prestel, Reduced stability of the Witt ring of a field and its Pythagorean closure, Amer. J. Math. 106 (1984), 1237-1260.

[Er] Yu. L. Ershov, Mapping a restriction of spaces of orderings of fields, Sibirsk. Mat. Zh. 27 (1986), 47-54; English transl., Siberian Math. J. 27 (1986), 181-187.

[J] G. J. Janusz, Algebraic number fields, Academic Press, New York, 1973.

[K] M. Krüskemper, On real local-global principles, preprint, Univ. of Münster, West Germany, 1988.

[L] T.-Y. Lam, The algebraic theory of quadratic forms, Benjamin, Reading, Mass., 1973 (revised printing, 1980).

[Le] D. B. Leep, Invariants of quadratic forms under algebraic extensions, preprint, Univ . of Kentucky, 1988.

[LW] D. B. Leep and A. R. Wadsworth, On the Hasse norm theorem mod $p$ (in preparation).

[OM] O. T. O'Meara, Introduction to quadratic forms, Springer-Verlag, New York, 1963.

[S] W. Scharlau, Quadratic and Hermitian forms, Springer-Verlag, Berlin, 1985.

[Se] J.-P. Serre, Local fields, Springer-Verlag, New York, 1979 (English transl. of Corps Locaux).

[SL] D. Shapiro and D. B. Leep, Piecewise equivalence of quadratic forms, Comm. Algebra 11 (1983), 183-217.

[STW] D. Shapiro, J.-P. Tignol, and A. R. Wadsworth, Witt rings and Brauer groups under multiquadratic extensions. II, J. Algebra 78 (1982), 58-90.

[W] A. Weil, Basic number theory, Springer-Verlag, New York, 1967.

Department of Mathematics, University of Kentucky, Lexington, Kentucky 40506

Department of Mathematics, University of California, San Diego, la Jolla, CaliforNIA 92093-0112 